\title{
DISCUSSION
}

\section{A semi-analytical solution for cylindrical cavity expansion in elastic-perfectly plastic soil under biaxial in situ stress field}

\author{
H. ZHOU*, G. KONG†, H. LIU* and S. L. CHEN†
}

\section{Contribution by S. L. Chen}

The authors revisited the interesting but challenging cavity expansion problem in elastoplastic soil subjected to anisotropic in situ stresses in the plane normal to the axis of the cylindrical cavity (Zhou et al., 2016). An approximate semi-analytical solution was developed by assuming incompressible material for the soil and by simply neglecting the shear stress and shear strain in the plastic zone. The addition of the displacement field for the plastic zone is claimed to be the major contribution of the paper over the already existing solutions for the stress and elastic-plastic interface originally derived by Galin (1946). However, there exist a number of limitations and shortcomings in the paper that require careful consideration.

The first issue is associated with the yield function form of the Tresca criterion, which was given in the paper (equation (17) in Appendix 1) as follows

$$
\left(\sigma_{y}-\sigma_{x}\right)^{2}+4\left(\tau_{x y}\right)^{2}=4 s_{\mathrm{u}}^{2}
$$

In writing the above equation, it is taken for granted that during the cavity expansion process the out-of-plane (vertical) principal stress $\sigma_{z}$ will always be the intermediate to the other two in-plane principal stresses

$$
\begin{aligned}
& \sigma_{1}=\frac{\sigma_{x}+\sigma_{y}}{2}+\sqrt{\left(\frac{\sigma_{x}-\sigma_{y}}{2}\right)^{2}+\tau_{x y}^{2}} \\
& \sigma_{3}=\frac{\sigma_{x}+\sigma_{y}}{2}-\sqrt{\left(\frac{\sigma_{x}-\sigma_{y}}{2}\right)^{2}+\tau_{x y}^{2}}
\end{aligned}
$$

Obviously, this is an overly simplistic assumption and not even correct for the axisymmetric cavity expansion problem. In the latter special case of $K=1$ ( $K$ denotes the ratio between the two in-plane in situ stresses), equation (17) may be strictly true (Hill, 1950) when the material is incompressible (Poisson ratio, $v=0 \cdot 5$ ) and when the initial stress state is fully isotropic, that is

$$
\sigma_{x, i}=\sigma_{y, i}=\sigma_{z, i}=\sigma_{0}
$$

where $\sigma_{x, i}, \sigma_{y, i}$ and $\sigma_{z, i}$ represent the three principal in situ stresses. It is readily anticipated that, for the anisotropic in situ stress condition, the relative magnitude of $\sigma_{z}$ with respect to $\sigma_{1}$ and $\sigma_{3}$ will inevitably be problem specific, and not necessarily remain the intermediate principal stress in general. From this point of view, the formulation of the paper is questionable, as the entire derivation is based on the

\footnotetext{
* College of Civil Engineering, Key Laboratory of New Technology for Construction of Cities in Mountain Area, Chongqing University, Chongqing, PR China.

$\uparrow$ College of Civil and Transportation Engineering, Key Laboratory of Ministry of Education for Geomechanics and Embankment Engineering, Hohai University, Nanjing, PR China.

+ Department of Civil and Environmental Engineering, Louisiana State University, Baton Rouge, LA, USA.
}

assumption that the yield function is expressible in terms of equation (17). One might argue that the close agreement between the semi-analytical solution and the Abaqus finite-element method (FEM) results in Figs 3 and 4 may be regarded as a convincing validation of the proposed analytical approach. It should be noted, however, that the comparisons shown in these two figures are pertinent only to the stress distributions (on the basis of Galin's solution, which is valid only when equation (17) holds true), yet in no way test the displacement solutions of equations (13a) and (13b) in the paper. Since the authors provided no detailed information regarding the in situ stress component $\sigma_{z, i}$ or the stress distributions of $\sigma_{z}$ in their Abaqus modelling, the discussion contributor would gather the calculated $\sigma_{z}$ turns out to be located in between the $\sigma_{\mathrm{r}}$ and $\sigma_{\theta}$ distributions in the plastic region for the two directions of $\theta=0$ and $\pi / 2$ considered so that equation (17) happens to apply. This can be seen more clearly if the authors present the distribution of $\sigma_{z}$ along the radial distance in addition to $\sigma_{\mathrm{r}}$ and $\sigma_{\theta}$ in Fig. 4 .

More serious is the authors' further assumption of zero shear stress in the entire plastic zone, which in combination with equation (5c) leads to $\mathrm{d} \varepsilon_{\mathrm{r} \theta}^{\mathrm{p}}=\varepsilon_{\mathrm{r} \theta}^{\mathrm{p}}=0$, and eventually $\varepsilon_{\mathrm{r} \theta}$ (equation (10)) for determining the displacement components. Such an assumption appears very much arbitrary and inappropriate, since the biggest challenge of taking into account the in situ stress anisotropy arises exactly from the involvement of the non-zero shear stress and shear strain in the plastic zone. Simply imposing $\tau_{\mathrm{r} \theta}$ and $\varepsilon_{\mathrm{r} \theta}$ to be trivial does not make any physical and logical sense. Although the authors provided the comparisons with the Abaqus simulation results in Figs 6 and 7 to verify the reliability of their semi-analytical displacement solutions, it is nevertheless by no means a complete and sound validation. This is because the 'good' agreement achieved in these two figures is under a special case of $\sigma_{\mathrm{a}}(10 \mathrm{MPa})$ being significantly larger than the in situ stress $\sigma_{0}(1 \mathrm{MPa})$. In this sense $\left(\sigma_{\mathrm{a}} \gg \sigma_{0}\right)$, it can be reasonably hypothesised that the anisotropic impact of the in situ stresses on the cavity response will be mostly masked and become negligible. And, conceivably, all the computed results should approach those corresponding to the case of an isotropic in situ stress field. It is thus no surprise that the approximate displacement solutions proposed by the authors compare broadly well with the FEM results (Figs 6 and 7). As a matter of fact, the calculated radial and tangential displacements $u_{\mathrm{r}}$ and $u_{\theta}$ exhibit little fluctuation with respect to the polar angle $\theta$, as is clearly seen in Fig. 7, which in turn justifies the hypothesis made above. The authors definitely need to conduct more refined validation analysis for more representative and comparable values of $\sigma_{\mathrm{a}}$ and $\sigma_{0}$, say $\sigma_{\mathrm{a}} / \sigma_{0}=4$. The discussion contributor's projection is that, under such circumstances, considerable discrepancies should take place between the approximate semi-analytical solution and the FEM simulations, owing to the relatively large effects of the shear stress and strain developed in the plastic zone. 
Finally, it seems that the statement after equation (36): 'Note that when the variable $r$ tends to infinity, the radial displacement $u_{\mathrm{r}}$ should tend to zero for such a cavity expansion problem' is not correct, because the plastic deformations are limited in an annulus region which put an upper bound on the $r$ value instead of infinity. Consequently, the two constants $A_{0}$ and $B_{0}$ need not be equal to zero. Similarly, there is no reason for the constant $J$ in equation (42) to vanish as ascertained by the authors.

\section{Authors' reply}

The authors are grateful to the discussion contributor for his comments, to which they respond as follows. As previous studies have not presented an analytical solution for cavity expansion under a biaxial in situ stress field, the solution presented here serves the important purpose of providing a set of benchmark results that could benefit from further, more sophisticated analysis. Unlike the FEM, the present solution can give predictions rapidly and without complex calculations for the plastic zone radius and the displacement in the plastic zone around the cylindrical cavity. An analytical solution such as the one presented in this paper can be used in tunnel lining design, for example, to investigate the problem of a circular cavity in an elastic-plastic soil. Previous elastic solutions for this problem were quite dated. Even so, no elastic-plastic solution had been presented for capturing the influence of the plastic behaviour of a medium on the displacement around the circular cavity. Therefore, the present solution completes the conventional analytical solutions for tunnel design problems. In particular, the solution can be used to calculate the displacement when a plastic zone occurs around the circular cavity, which could previously be investigated only using the FEM. Another application for using the proposed solution is the interpretation of pressuremeter tests under a biaxial in situ stress field (Bernier et al., 2007; Verstricht et al., 2010). Additionally, the closed-form solution proposed here could be used to back-calculate the stress ratio $K$ for the biaxial in situ stress field.

The discussion contributor proposed two questions about the authors' analytical solution. The first issue is the use of the Tresca criterion (equation (17) in Appendix 1) in deriving the analytical solution. In fact, it is very difficult to find an explicit solution for the cavity expansion under a biaxial stress field because the kinematics are non-cylindrically symmetric, which is different from the conventional cylindrical cavity expansion. In this case, a hypothesis that the vertical stress $\sigma_{z}$ is assumed as the intermediate stress is used, although this cannot be applied to all the stress conditions in geotechnical engineering. An explicit solution for the displacement in the plastic zone can be found based on this hypothesis. This solution has theoretical value although it is not suitable for the case that the intermediate stress occurs in the cavity expansion plane. The discussion contributor had some doubts about the lack of FEM validation of the displacement solutions of equations (13a) and (13b). Actually, Figs 6 and 7 in the paper compared the theoretical displacement predictions with the FEM results. Therefore, the FEM results can be regarded as a convincing validation of the presented analytical solution.

Another issue is the assumption of the zero shear stress in the entire plastic zone. Because a uniform pressure (no surface traction) is applied at the cylindrical cavity wall, the shear stress near the cavity wall (plastic zone) is small and can be neglected. It should be noted that the investigated problem is a pressure-controlled cavity expansion problem and that the shape of the cylindrical cavity changes during

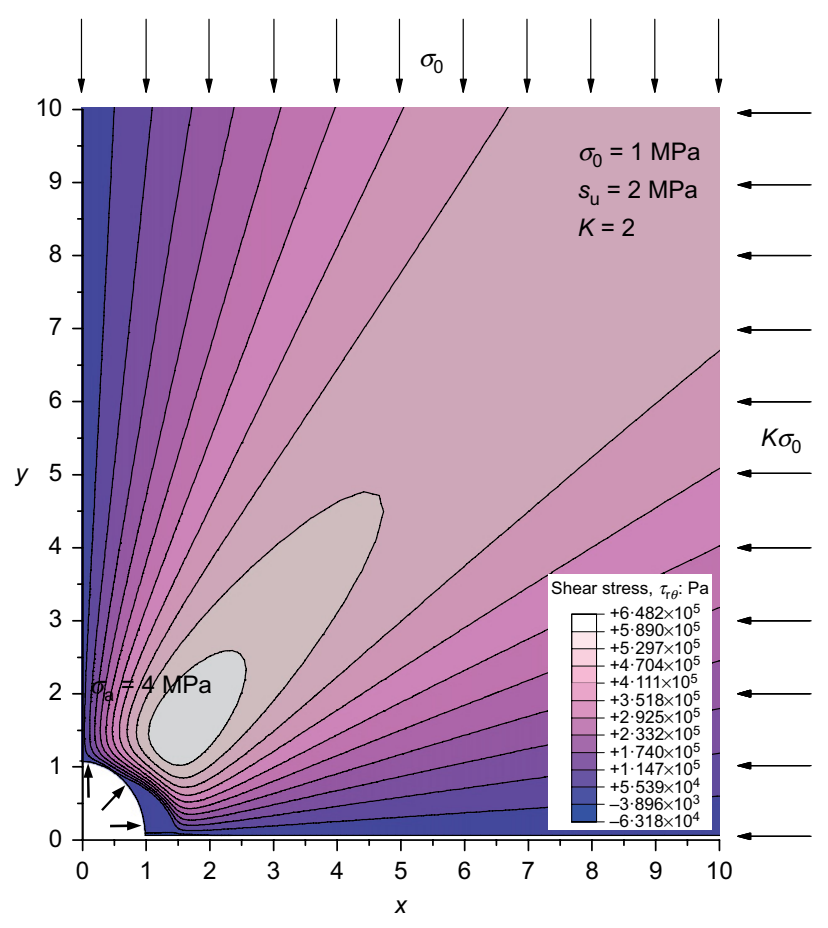

Fig. 10. FEM results for the shear stress contours around the cylindrical cavity for the case of $\sigma_{\mathrm{a}} / \sigma_{0}=4$

the cavity expansion process. Such a cavity change impedes the formation of shear stress. However, if the cavity expansion is displacement-controlled so that the cylindrical cavity shape does not change during the expansion process, the value of the shear stress in the plastic zone may be large and cannot be neglected. For the proposed problem in the paper, the assumption is reasonable and the FEM results confirm this point. In addition, the discussion contributor believed that the 'good' agreement achieved in the two figures (Figs 6 and 7 in the paper) is under a special case of $\sigma_{\text {a }}$ $(10 \mathrm{MPa})$ being significantly larger than the in situ stress $\sigma_{0}$ (1 MPa). However, the authors conducted a FEM analysis for the case of $\sigma_{\mathrm{a}} / \sigma_{0}=4$ and the shear stress contours around the cylindrical cavity are plotted in Fig. 10. It can be found that the shear stress in the plastic zone varies from $-0.06 \mathrm{MPa}$ to $0.05 \mathrm{MPa}$, which is relatively small compared to the value of in situ stress. It implies that the shear stress in the plastic zone is small even under the condition of small ratio of $\sigma_{\mathrm{a}} / \sigma_{0}$. In other words, the assumption of the zero shear stress in the entire plastic zone is reasonable.

Finally, the discussion contributor stated that the sentence after equation (36): 'Note that when the variable $r$ tends to infinity, the radial displacement $u_{\mathrm{r}}$ should tend to zero for such a cavity expansion problem' is not correct. It should be noted that the radius of the plastic zone can be expressed as follows (equation (3) in the paper)

$$
r_{\mathrm{b}}(\theta)=\frac{c_{0}(1+\beta)}{\sqrt{1+\left[(1+\beta / 1-\beta)^{2}-1\right] \sin ^{2} \theta}}
$$

where

$$
c_{0}=a \times \mathrm{e}^{1 / 2 s_{\mathrm{u}}\left[-(1+K) \sigma_{0} / 2+\sigma_{\mathrm{a}}-s_{\mathrm{u}}\right]}
$$

The radius of the plastic zone has an exponential relation with the cavity wall pressure $\sigma_{\mathrm{a}}$ and thus the radius of the plastic zone increases rapidly with increasing cavity wall pressure. The radius of the plastic zone tends to infinity when the cavity wall pressure is very large. In this case, 
the statement after equation (36) is correct. If the two constants $A_{0}$ and $B_{1}$ are not equal to zero, the calculated displacement will tend to infinity, which is not correct. Similarly, the constant $J$ in equation (42) should also be equal to zero.

\section{REFERENCES}

Bernier, F., Li, X. L. \& Bastiaens, W. (2007). Twenty-five years' geotechnical observation and testing in the Tertiary Boom clay formation. Géotechnique 57, No. 2, 229-237, http://dx.doi.org/ 10.1680/geot.2007.57.2.229.
Galin, L. A. (1946). The plane elastoplastic problem: plastic regions near round holes in plates and beams. J. Appl. Math. Mech. 10, No. 3, 367-386.

Hill, R. (1950). The mathematical theory of plasticity. Oxford, UK: Oxford University Press.

Verstricht, J., Areias, L., Bastiaens, W. \& Li, X. L. (2010) Measurement techniques for in situ stresses around underground constructions in a deep clay formation. EPJ web of conferences, vol. 6, paper no. 22018. Paris, France: EDP Sciences.

Zhou, H., Kong, G. \& Liu, H. (2016). A semi-analytical solution for cylindrical cavity expansion in elastic-perfectly plastic soil under biaxial in situ stress field. Géotechnique 66, No. 7, 584-595, http://dx.doi.org/10.1680/jgeot.15.P.115. 